\title{
Retinal Adaptation of Visual Processing Time Delays
}

\author{
D. M. WOLPERT,* R. C. MIALL,* B. CUMMING,* S. J. BONIFACE $\dagger$ \\ Received 20 July 1992; in revised form 5 November 1992
}

\begin{abstract}
A significant proportion of the processing delays within the visual system are luminance dependent. Thus placing an attenuating filter over one eye causes a temporal delay between the eyes and thus an illusion of motion in depth for objects moving in the fronto-parallel plane, known as the Pulfrich effect. We have used this effect to study adaptation to such an interocular delay in two normal subjects wearing $75 \%$ attenuating neutral density filters over one eye. In two separate experimental periods both subjects showed about $60 \%$ adaptation over 9 days. Reciprocal effects were seen on removal of the filters. To isolate the site of adaptation we also measured the subjects' flicker fusion frequencies (FFFs) and contrast sensitivity functions (CSFs). Both subjects showed significant adaptation in their FFFs. An attempt to model the Pulfrich and FFF adaptation curves with a change in a single parameter in Kelly's [(1971) Journal of the Optical Society of America, 71, 537-546] retinal model was only partially successful. Although we have demonstrated adaptation in normal subjects to induced time delays in the visual system we postulate that this may at least partly represent retinal adaptation to the change in mean luminance.
\end{abstract}

Pulfrich effect Adaptation Latency Flicker Depth perception

\section{INTRODUCTION}

When a moving target is viewed with a neutral density filter placed over one eye, it appears displaced in depth. This is known as the Pulfrich effect and is usually described with regard to a pendulum swinging in a fronto-parallel plane in front of an observer (Pulfrich, 1922). Pulfrich credits Fertsch with the suggestion that the reduced light levels reaching the covered eye could delay the neural signal. The attenuated eye therefore reports a delayed signal of the moving target and a retinal disparity is generated between the two eyes resulting in an illusion of depth. With a filter over the right eye, as in Fig. 1, the pendulum is seen to rotate elliptically anti-clockwise (as if viewed from above).

Psychophysical studies have since supported Fertsch's suggestion and have confirmed that visual processing time increases with reduced stimulus intensity (Lit, 1949; Julesz \& White, 1969; Rogers \& Anstis, 1972; Morgan $\&$ Thompson, 1975). Recordings from the visual cortex of cats have also confirmed this relationship (Cynader, Gardner \& Douglas, 1978; Carney, Paradiso \& Freeman, 1989). Recordings from cat and monkey retinal ganglion cells have provided evidence for a peripheral site of this intensity-dependent response (Lennie, 1981; Bolz, Rosner \& Wassle, 1982); the delay being probably due to changes in photoreceptor behaviour (Baylor \&

*University Laboratory of Physiology, Parks Road, Oxford OXI 3PT, England.

†Department of Clinical Neurophysiology, Radcliffe Infirmary, Woodstock Road, Oxford, England.
Hodgkin, 1973; Mansfield \& Daugman, 1978; Drum, 1984). Thus, the attenuating filter worn over one eye induces a delay by reducing light intensity and increasing photoreceptor latencies.

We have been interested in adaptation to time delays in the visuo-motor system. There is evidence that monkeys can partially adapt their oculomotor smooth pursuit system to changes in the oculomotor feedback delay (Deno, Keller \& Crandall, 1989). We were interested to test whether the perceptual system could, on its own, adapt to changes in time delay. We therefore used the Pulfrich phenomenon in order to examine whether normal subjects can adapt to a monocular increase in visual latency. Flash and pattern reversal visual evoked responses (VERs) were also recorded. We have also attempted to distinguish between a retinal and a cortical site for adaptation by measuring flicker fusion frequencies (FFFs) in the attenuated eye over the course of adaptation. A previous study reported changes in the magnitude of the Pulfrich effect over a 5 day adaptation period during which subjects wore $80 \%$ attenuating filters (Douthwaite \& Morrison, 1975). They reported no change in the FFF measured at one contrast level. It is not possible to estimate the change in FFF expected from a change in intraocular delays, as determined by the Pulfrich phenomenon, nor from changes in the latency of visual evoked potentials (VEPs). We therefore simulated Kelly's (1971) model of visual processing (see Appendix) to see whether the model could explain both our observed change in interocular delays and in FFFs. From the simulation and from our own results, the 


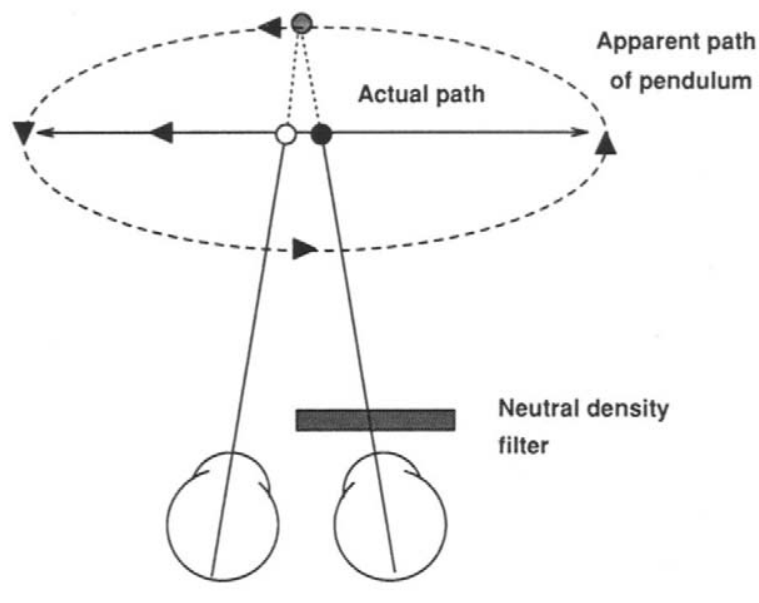

FIGURE 1. Pulfrich effect of a pendulum swinging in the fronto-parallel plane, viewed from above. The empty circle is the actual bob, the solid circle represents the delayed bob position that the attenuated right eye sees during leftward movement, and the shaded circle represents the perceived position of the bob. The apparent path of the bob then falls on an ellipse.

expected changes in the FFFs would be small $(c .5 \mathrm{~Hz})$. It is unclear whether the method used by Douthwaite and Morrison (1975) was sufficiently precise to detect such small changes. We therefore investigated this question again using forced-choice paradigms to determine both the FFFs and the magnitude of the Pulfrich effect. The response of ganglion cells to flicker has been studied in the cat; Enroth (1952) showed that the ganglion cells generated bursts of spikes phase-locked to the flicker waveform up to the fusion frequency. Above this threshold the pulse intervals become random and are no longer correlated with the stimulus frequency. Therefore it is reasonable to conclude that the FFF is limited by the retina and so an increase in the FFF during an adaptive period is likely to be a result of retinal changes. Since flicker fusion depends upon contrast, changes in the FF measured at any one contrast may result from changes only in contrast sensitivity. To exclude such effects, we also measured spatial and temporal contrast sensitivity functions (CSFs).

\section{METHODS}

Two subjects wore a neutral density filter over one eye for two experimental periods separated by a 1 month gap. The first period lasted 9 days. Both subjects wore $75 \%$ attenuating neutral density filters ( 2 stops, $0.6 \mathrm{ND}$ ) over one eye throughout the day; the patched eyes were taped shut at night. The filters were set within a window cut from a black eye patch, thus excluding all light except that transmitted by the filter. VERs were measured on day 0 and day 8 of this period. The second period lasted 11 days; subject 1 wore a $70 \%$ attenuating neutral density filter contact lens (Lunelle) while subject 2 again wore a $75 \%$ attenuating neutral density filter eye patch. During this second period we also measured FFFs in the attenuated eye and spatial and temporal contrast sensitivities. During both periods we estimated the delay in the filtered eye relative to the normal eye by means of the Pulfrich effect.
Estimating interocular delay using the Pulfich effect

The Pulfrich effect was simulated on a computer screen viewed through a Wheatstone stereoscope. The subjects sat in a darkened room $290 \mathrm{~cm}$ in front of the display. White vertical lines, length $5 \mathrm{~cm}$, on a grey background were presented separately to each eye to produce a stereo image. Both lines moved to and fro horizontally at $1 \mathrm{~Hz}$, with constant specd $(3.28 \mathrm{deg} / \mathrm{sec})$ and an excursion of \pm 0.82 deg about a central fixation marker. Anti-aliasing was used to achieve sub-pixel resolution on the screen. The luminance of the screen was $26.7 \mathrm{~cd} \mathrm{~m}^{2}$; the contrast was $50 \%$. At the moment of reversal of target direction there will be a period during which the eyes will see target motion in different directions; this could generate the perception of motion in depth independent of the change in retinal disparity (Regan, Beverley \& Cynader, 1979). To avoid this the screen was blanked for $60 \mathrm{msec}$ at the moment of reversal of the stimulus motion.

On each trial a fixed horizontal disparity was introduced between the vertical lines. The sign of the disparity was reversed for the two directions of motion inducing an illusion of a fixed depth between rightward and leftward trajectories for a given disparity. The subject's task was to fixate on the central marker and to estimate the direction of rotation of the stimulus. The apparent disparity caused by the filter could thus be nulled by a fixed disparity between the moving lines displayed to each eye.

A two-alternative forced-choice paradigm (2AFC) was then used to determine the "null" disparity at which no change in depth was perceived between the rightward and leftward trajectories. At each session 6 different disparities, spread equally about each subject's mean null disparity, were presented in a pseudo-random order for 40 trials each. The null disparity was then scaled by stimulus velocity to give a measure of interocular time delay. Thus estimated interocular time delay was calculated as $d / v \mathrm{sec}$, where $d$ is the null disparity and $v$ is the target velocity. We measured these estimates of the interocular time delay prior to filter placement, daily during the adaptation phase and after removal of the filter. On removal of the filter the interocular time delay was estimated immediately and then at 1, 4, 22 and $96 \mathrm{hr}$ in the first experimental period and at 24 and $48 \mathrm{hr}$ in the second experimental period.

To check whether adaplation was specific to the test velocity used we examined the effect of stimulus velocity on our estimates of interocular delay after adaptation (on day 8 of the first experimental period) using velocitics 50\% smaller and 50\% larger than the standard velocity. These stimulus velocities had previously not been seen by either subject. The frequency of target motion was adjusted to maintain a fixed amplitude throughout.

\section{Pulfrich effect data analysis}

A cumulative Gaussian function was fitted to the forced-choice data to calculate the mean. The standard 
error of the mean was estimated from the $95 \%$ fiducial limits of the psychometric function. The fiducial limits were estimated using the method of Watson and Pelli (1983). The data was plotted as the mean interocular delay $\pm 1 \mathrm{SE}$ over the duration of the experiment. Regression lines were fitted to the adaptation portions of the plot to estimate the rate of adaptation. A multivariate probit analysis of velocity and disparity was used to estimate the effects of the different velocity targets on our measurements.

\section{Flicker fusion frequencies}

During the second adaptation period we also measured FFFs to a sinusoidally flickering $5 \mathrm{~mm}$ diameter yellow light emitting diode (LED), viewed through an artificial pupil of $2.5 \mathrm{~mm}$ diameter. The luminance of the LED was $1800 \mathrm{~cd} \mathrm{~m}^{-2}$ (8836 td). To ensure a linear intensity curve, we used a pulse width modulation circuit: since the emitting energy is proportional to the LED pulse duration this overcomes the problems of the non-linear relationship of LED intensity to input voltage. The LED was pulsed at $1 \mathrm{kHz}$; the duration of each pulse was determined by a sinusoidal modulating voltage. This modulating voltage was generated from a computer and output through a D/A channel at $16 \mathrm{kHz}$.

The LED was fixed $30 \mathrm{~cm}$ from the subject's eye; chin and forehead rests and an alignment ruler were used to ensure a constant position of the eye relative to the LED for the duration of the experiment. The room was dimly illuminated by an $8 \mathrm{~W}$ high frequency fluorescent tube $(20 \mathrm{kHz})$.

The intensity of light, $f(t)$, seen by the eye at time $t$ is given by:

$$
f(t)=L(1+m \sin (\omega t)
$$

where $L$ is the background illumination, $m$ is the modulation of the LED and $\omega$ is its frequency of oscillation. We measured the FFF at three values of $m$ for each subject during the course of the experiment ( $m=0.20$, 0.28 and 0.84 ). An interval two-alternative forced-choice paradigm was used. On each trial the LED was presented for two $400 \mathrm{msec}$ periods, separated by a gap of $100 \mathrm{msec}$. The LED flickered during one interval, and had constant luminance $L$ for the other interval. In each session, six different frequencies and three values of $m$ were tested, each presented 40 times in a pseudorandom order.

A cumulative Gaussian function was fitted to the forced-choice data to determine the $75 \%$ threshold along with the SE on this threshold.

\section{Visual evoked responses}

During the first experimental period we recorded visual evoked responses. Each eye was tested separately. The testing was performed in two sessions, one on day 0 before and after placement of the filter and on day 9 before removal of the filter.

VERs were recorded to both a pattern reversal stimulus (mechanical moving mirror checkerboard, Digitimer DI12) and to a photopic flash stimulus. The VER was VR $3310 \%$ recorded between two electrodes; one on the occiput $\left(\mathrm{O}_{7}\right)$ and the other on the vertex $\left(\mathrm{C}_{z}\right)$. The flash VERs were recorded to a photopic flash presented at $1 \mathrm{~Hz}$ for 128 repetitions (Grass stimulator PS22, luminance $375 \mathrm{k}$ candle power). The pattern reversal stimulus was centred on the fovea subtending $17.4 \mathrm{deg}$ at the eye; the pattern subtended $48 \mathrm{~min}$ arc. The mean luminance was $585 \mathrm{~cd} \mathrm{~m}^{-2}$ with a contrast of $85 \%$. For each trial the stimulus was presented 128 times at $1 \mathrm{~Hz}$; each trial was repeated twice. On day 0 recordings were made under three conditions; with no filter, 75 and $94 \%$ attenuating filters. On day 9 recordings were made under two conditions; 75 and $94 \%$ filter.

For each condition the recordings from the 128 stimulus presentations were averaged. For the flash VERs the $P_{100}$ latency was measured, and the latency of the first major positive deflection was measured for the pattern reversal VER.

\section{Contrast sensitivities}

We measured the spatial and temporal CSFs of both eyes to a sinusoidal grating on day 8 of the second period of adaptation and again 3 days after removal of the filter. To equalize light levels a filter (or contact lens) and a $2.5 \mathrm{~mm}$ diameter artificial pupil was worn on both eyes for all measurements.

Subjects sat $2.5 \mathrm{~m}$ in front of a multisync computer monitor on which was displayed horizontal yellow and black gratings $(14 \times 17.5 \mathrm{~cm}, 3.2 \times 4.0 \mathrm{deg}$; the mean luminance of the stimulus was $85 \mathrm{~cd} \mathrm{~m}^{2}$ ). We measured contrast sensitivities for six different spatial frequencies $(0.5-20 \mathrm{c} / \mathrm{deg})$ and for six different temporal frequencies (1-20 Hz sinusoidal flicker for a $2 \mathrm{c} / \mathrm{deg}$ grating). A spatial two-alternative forced-choice paradigm was employed using a staircase procedure; at each stimulus presentation a grating was displayed either on the right or left side of the screen. The contrast was reduced by a factor of 0.15 for a correct response and increased by a factor of 2.0 for an incorrect response (Cambridge Research Systems Ltd). Each spatial and temporal frequency was presented 50 times.

The results from the contrast sensitivity studies were analysed by probit analysis to produce estimates of the $75 \%$ correct response rate and SEs on this estimate. The contrast sensitivities were plotted against spatial and temporal frequency on a $\log \log$ graph.

\section{RESULTS}

\section{Interocular delay}

The time-course of adaptation to filter placement as measured by the two-alternative forced-choice paradigm is shown in Fig. 2. The results below refer to the first experimental period; the second experimental period was essentially similar to the first. Prior to filter placement neither subject's interocular time delay was significantly different from zero at the $1 \%$ level. On placement of the filters the estimated interocular time delay induced was $10.9 \pm 0.76 \mathrm{msec}$ (one SE) for subject 1 and 

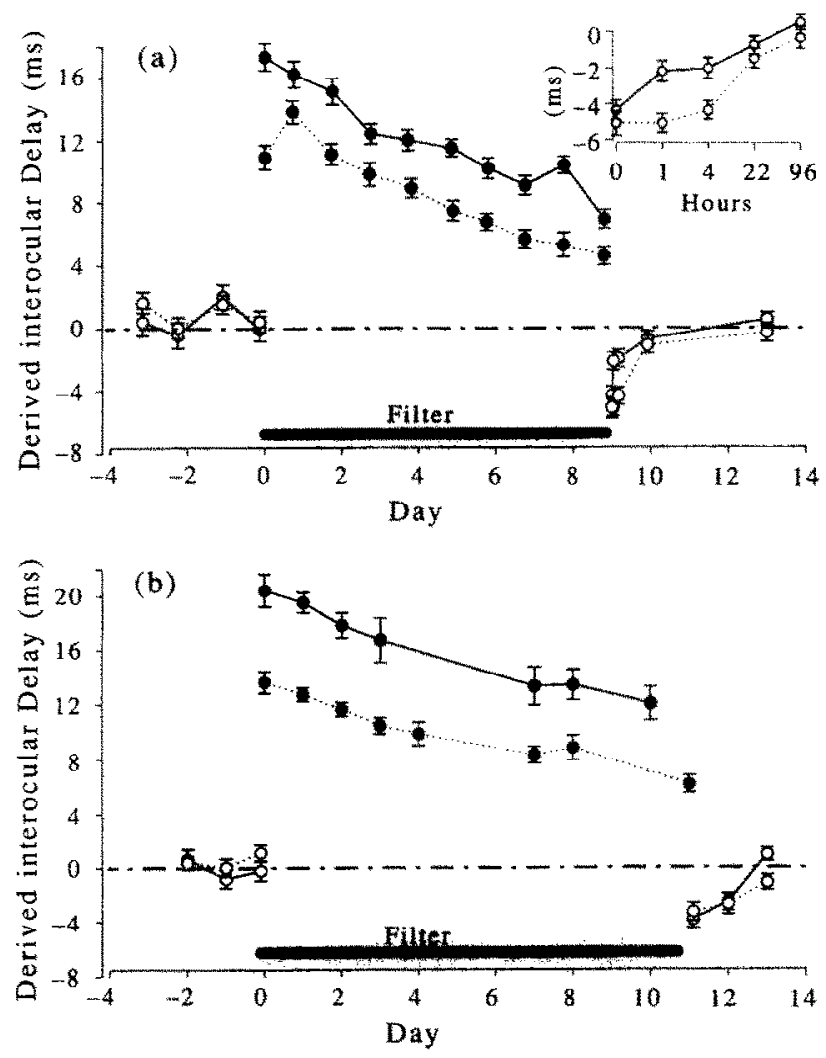

FIGURE 2. Plot of derived interocular delay against time for (a) first experimental period and (b) second experimental period. Subject 1 (solid line \pm 1 SE), wore the fitter on his right eye while subject 2 (dashed line $\pm 1 \mathrm{SE}$ ) wore the filter on his left eye; positive values of interocular delay represent delay in the attenuated eye. Hollow circle are measurements made without a filter and the solid circles are those made with the filter in place. The inset in (a) shows the interocular delay against hours after filter removal for the first experimental period on an expanded time base (note the time axis is nonlinear).

$17.3 \pm 0.87 \mathrm{msec}$ for subject 2 . These results are significantly different from the pre-filter $(P<0.0001)$. During the subsequent 9 days both subjects showed significant adaptation with the delays falling by $59-60 \%$ to $4.5 \pm 0.56 \mathrm{msec}$ for subject 1 and $6.9 \pm 0.61 \mathrm{msec}$ for subject 2. A linear regression of interocular delay against time during this adaptation period was significant for each subject $\left(P<0.0001, n=10, r^{2}=0.90\right.$ and 0.92$)$. The rate of adaptation estimated from the regression analysis was $1.04 \mathrm{msec} /$ day for subject 1 and $0.95 \mathrm{msec} /$ day for subject 2 .

On removal of the filters, reciprocal delays of $5.1 \pm 0.66 \mathrm{msec}$ and $4.3 \pm 0.56 \mathrm{msec}$ were measured. The overshoot was statistically significant $(P<0.01)$ at 0,1 and $4 \mathrm{hr}$ after removal of the filter for the first experimental period (inset, Fig. 2) and at 0 and $24 \mathrm{hr}$ for the second experimental period; readaptation was complete within $96 \mathrm{hr}$.

The multivariate probit analysis showed that the stimulus velocity did not have a significant effect at the $5 \%$ level for either subject.

\section{Flicker fusion frequencies}

Our measurements of FFFs were not as smooth as those of the Pulfrich effect. The FFF measurements were more variable from trial to trial and both subjects found this task more taxing than the Pulfrich test. Figure 3 shows the FFFs recorded for three modulation values $m$ over the 11 day adaptation period. Immediately upon placement of the filter the FFFs fell significantly for both subjects at all $m$ values. Over the subsequent 11 days the FFFs increased significantly for all three $m$ values and for both subjects. On removal of the filters on day 11 neither subject had a statistically significant overshoot in flicker frequencies (although subject 2 did show a small overshoot). Two days after removal of the filter the FFFs were back to pre-filter values; replacement of the filter at this stage showed the same effect as the initial placement of the filter (see the right most points in each curve in Fig. 3).

\section{Visual evoked responses}

On placement of the $75 \%$ filter the latency of the $P_{10 n}$ peak in the pattern reversal visual evoked response increased by $4 \mathrm{msec}$ for subject 1 and $5 \mathrm{msec}$ for subject 2. There was no measurable decrease in the latency when the VERs were next recorded on day 8 . There was no significant change in the latency for the flash VER under any of the conditions tested. However, the flash stimulus was so bright that the latency differences would be minimal between the attenuated and unattenuated states.

\section{Contrast sensitivities}

Figure 4 shows the CSFs measured under three conditions. The results demonstrate that there was no significant difference between the contrast sensitivities of the two eyes after adaptation or between the patched eye in the normal or adapted state.

\section{RETINAL MODEL}

As the FFFs showed adaptation to a long term change in luminance and the FFFs are limited by retinal processes, there is a possibility that the adaptation to the Pulfrich effect is secondary to changes in the retina. We therefore attempted to model both the changes in FFF and in the Pulfrich effect using retinal changes only.

Kelly (1971) has produced a model which accounts for both the high and low frequency flicker responses of human subjects. The model has two components which are taken to represent biochemical and neural properties of the retina. The firsi represents a photoresponsive diffusion process (although the underlying physiological process is unclear), which is followed by a second non-linear component representing certain retinal pathways and synapses of the plexiform layer. Figure 5 shows the results of the model simulations; the details of the model and simulations are given in the Appendix. Adaptation of the Pulfrich phenomenon and the FFFs was qualitatively fitted by a change in a single parameter, $\tau$. However, it appears that the observed FFF adaptation curves differed from the changes in the model in two respects. First the time-course of adaptation appears to be non-linear (Fig. 3). Second, there was little or no 
(a)

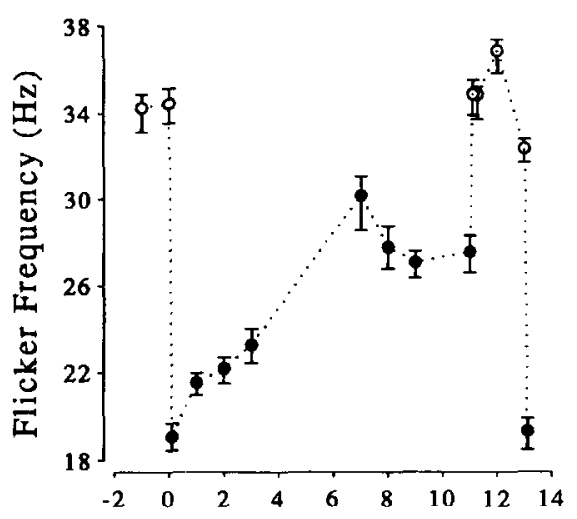

(d)

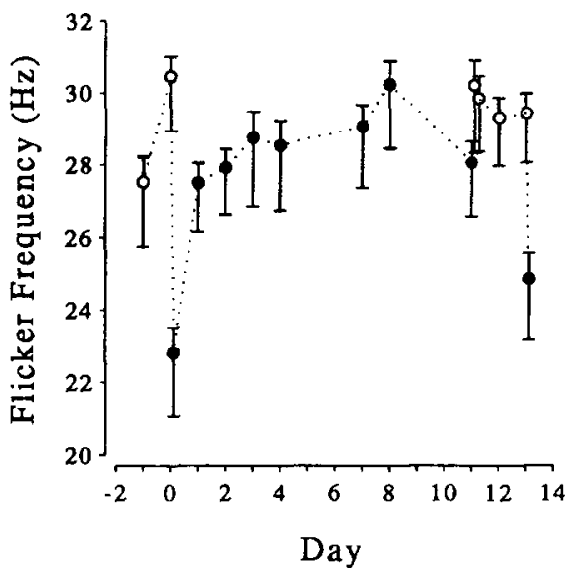

(b)

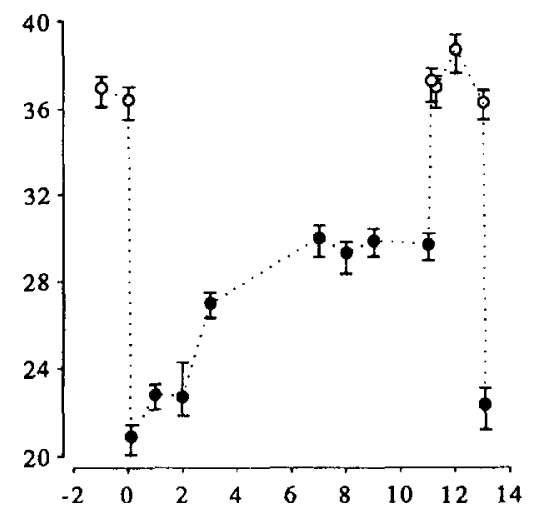

(e)

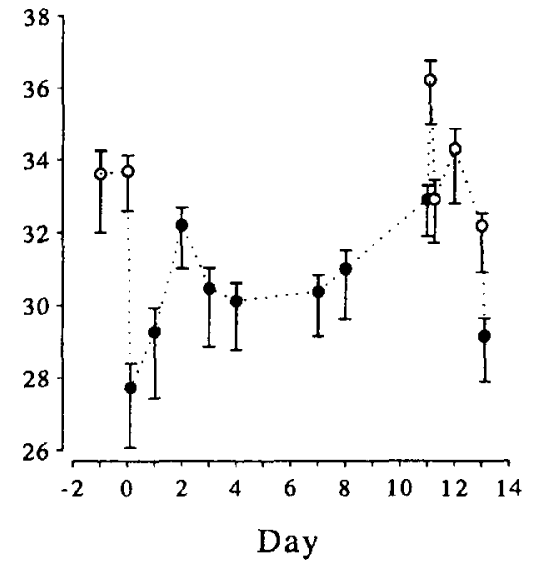

(c)

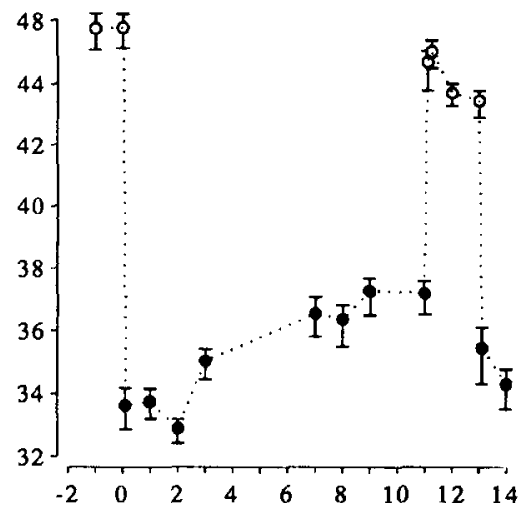

(f)

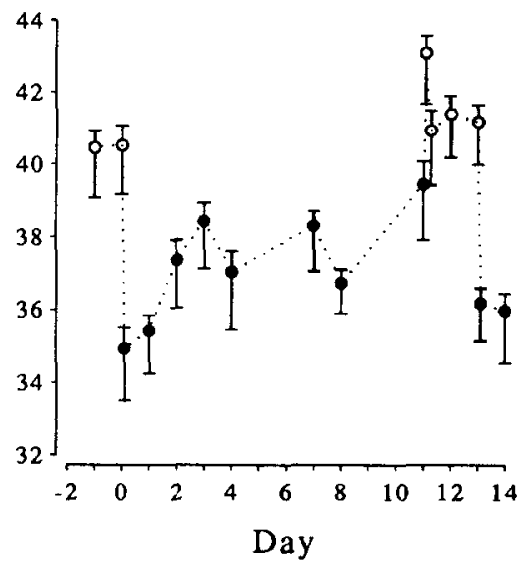

FIGURE 3. Plot of FFFs against time. (a)-(c) Subject 1 at $m$ values of $0.20,0.28$ and 0.84 respectively. (d)-(f) Subject 2 for the same $m$ values. The open circles are measurements made without the filter and the solid circles are measurements made with the filter. Error bars are $1 \mathrm{SE}$ about the $75 \%$ correct response rate as calculated from the probit analysis.

overshoot in FFF on removal of the filters, whereas the model showed significant overshoot (Fig. 5).

\section{DISCUSSION}

A light attenuating filter placed over one eye causes a delay in monocular transmission. We have measured long term adaptation to this delay using the Pulfrich effect. The percentage adaptation seen over a 9 day period was similar for both subjects $(60 \%$ for subject 1 and $59 \%$ for subject 2). Adaptation was surprisingly slow at the rate of about $1 \mathrm{msec} /$ day and was close to linear with $r^{2}>0.9$ for both subjects. We do not believe that this effect can be related to learning some aspects of the task for two reasons. First, the rate of adaptation was similar in the two separate experimental periods, and second the adaptation continued unabated during the 4 days subject 1 and 3 days subject 2 were not tested during the second period (see Fig. 2). The adaptation was not specific to the test velocity as shown by the different target velocities tried on day 8 of the first period. This confirms and extends the findings of Douthwaite and Morrison (1975).
It has been known for over 100 years that reaction times are inversely related to stimulus intensity (Cattell, 1886), but it was not until relatively recently that the site of this delay was isolated. The luminance dependent delay occurs in the phototransduction process in the retina (Baylor \& Hodgkin, 1973; Mansfield \& Daugman, 1978; Drum, 1984; Lamb \& Pugh, 1992). Thus the cause of the interocular delay in the Pulfrich effect is well established, but it is less clear where the site of adaptation may be.

We have also demonstrated adaptation in FFFs in the attenuated eye over the same period. The fall in FFFs on placement of the filter on day 0 was expected as they are known to change with luminance levels (e.g. Kelly, 1972). However over the next 12 days the FFFs rose to near pre-filter values for subject 2 and the adaptation was about $50 \%$ complete for subject 1 . It has not been previously shown that the FFFs adapt over a period of days to a new level. Little overshoot was seen for either subject after removal of the filter. The change in FFF is unlikely to be due to learning, as after a de-adaptation period of only 2 days (without the filter) the effect of filter replacement was as great as it had been on the first day. Had there been a significant learning effect, we 

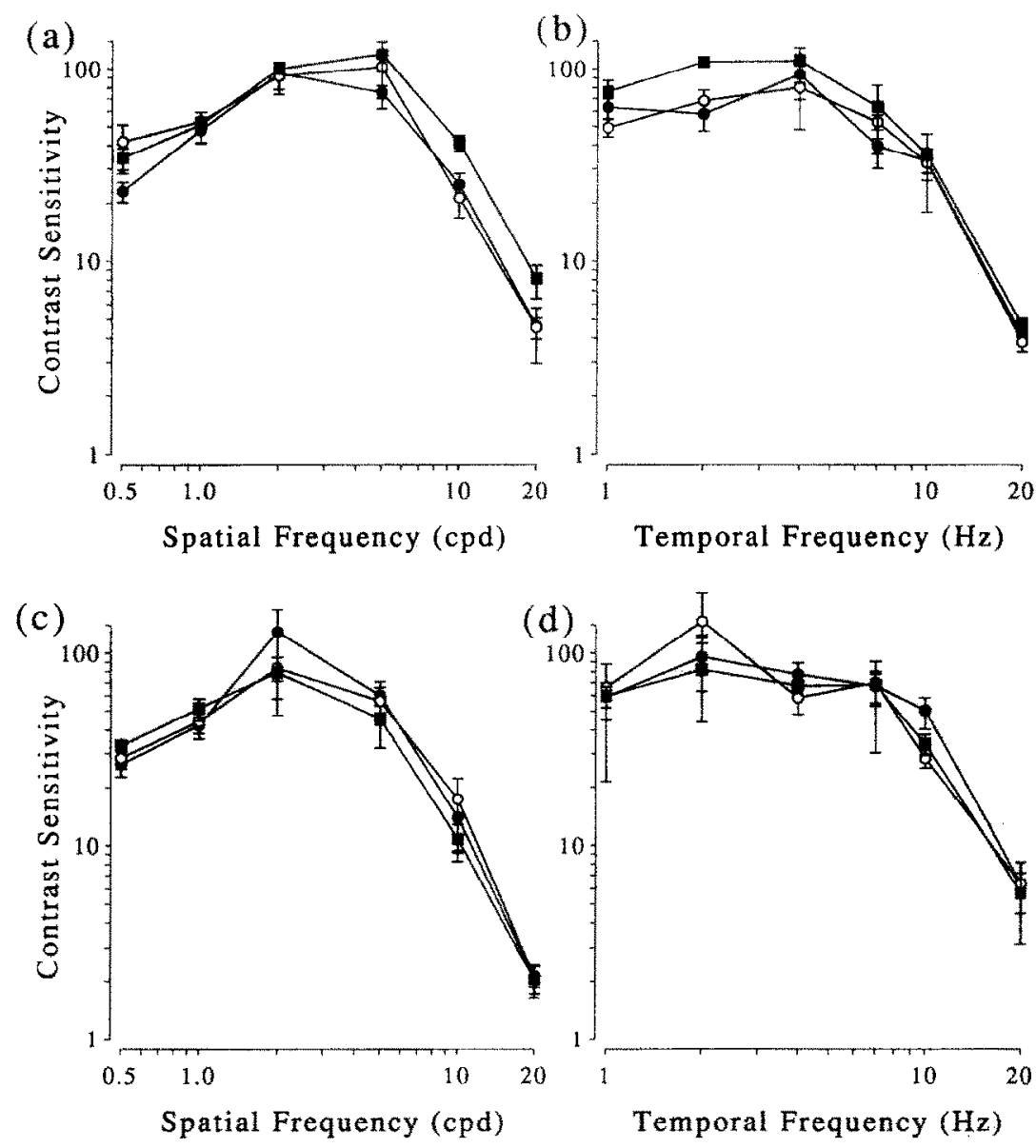

FIGURE 4, Plots of (a) spatial and (b) temporal CSFs for subject 1. (c) Spatial and (d) temporal CSFs for subject 2. The open circles are measurements for the unpatched eye and the solid circles for the patched eye (both measured on day 8 ). The solid squares are measurements from the previously patched eye 3 days after filter removal. All measurements were made with a filter over the tested eye and with a $2.5 \mathrm{~mm}$ diameter artificial pupil. Error bars represent $1 \mathrm{SE}$.

would have expected a much smaller drop in FFF on replacement of the filters.

Although the time-course of adaptation was similar for both our subjects the absolute change in interocular delay and in the FFFs was very different (but consistent) between subjects. We suppose that these differences imply different inherent processing delays between the subjects. As is common in adaptive studies the rate of re-adaptation was much faster than the original adaptation. Adaptation to the Pulfrich effect and in the FFFs took more than 9 days whereas re-adaptation after removal of the filter took less than 2 days.

Our data showed adaptive changes in FFFs at three different contrast levels. Although this could be due to changes in the temporal properties of retinal processing, the same results could be explained by a shift in the subjects' CSFs. To exclude this we measured the spatial and temporal CSFs. These showed no significant change between the adapted and de-adapted states; we can therefore exclude this as a possible mechanism for the change in FFFs.

This result differs from that found by Douthwaite and Morrison (1975) who found no changes in the FFF. One reason for this may be that the magnitude of the adaptive change is rather small-over the first five days we found changes in the FFFs of between 2 and $6 \mathrm{~Hz}$. Such changes would have been difficult for Douthwaite and Morrison to detect as they did not use a forcedchoice method. Their FFF measurements were "made using descending runs" (presumably a method of adjustment). No attempt was described to randomize any of the stimulus parameters, and although each recording of the FFF was based on 10 measurements no statistical analysis was performed. Thus Douthwaite and Morrison concluded that the adaptation they saw to the Pulfrich effect was perceptual and not retinal.

No significant changes were seen in the VER latencies over the adaptation period despite the $4-5 \mathrm{msec}$ increase on placement of the filter. However, the VER techniques cannot reliably detect time delays as small as $2 \mathrm{msec}$ which might be expected if the VER showed the same percentage change in latency as measured in the psychophysical tests over the 9 day adaptation period.

We believe that our data suggest that the adaptive sites include the retina. Simulations of a model of the human retina can qualitatively account for the reduction in interocular delay with concomitant overshoot on removal of the filter (like our results the overshoot is smaller than the adaptation). For the flicker data the model demonstrates a rise in frequency with adaptation 
(a)

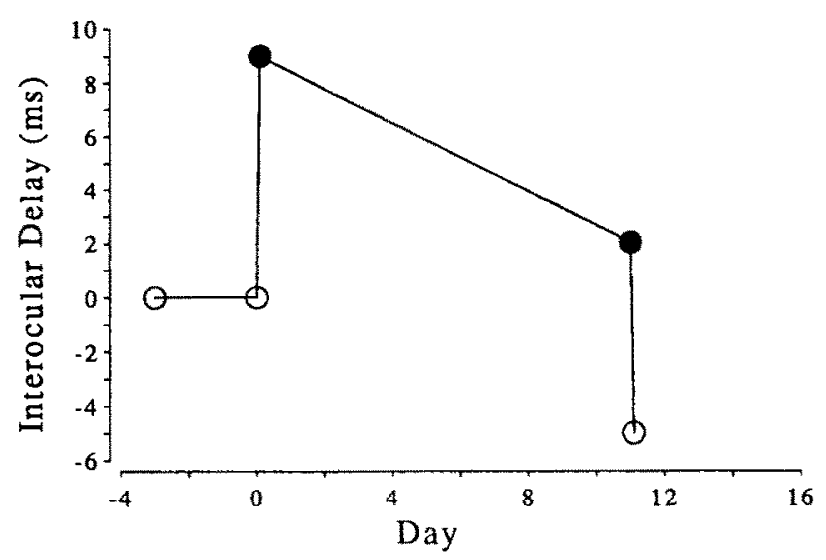

(b)

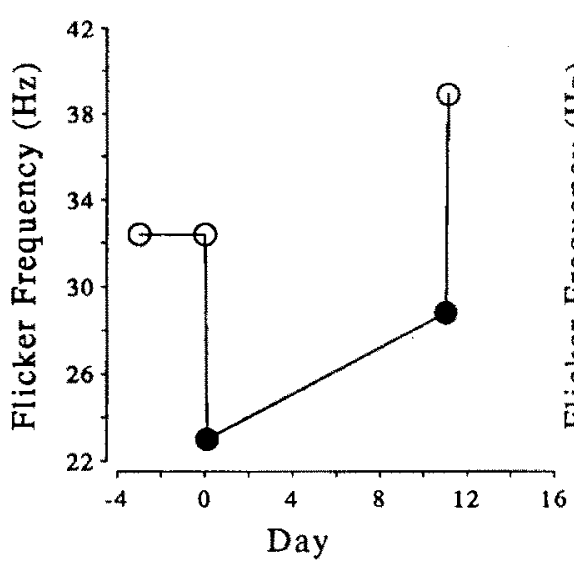

(c)

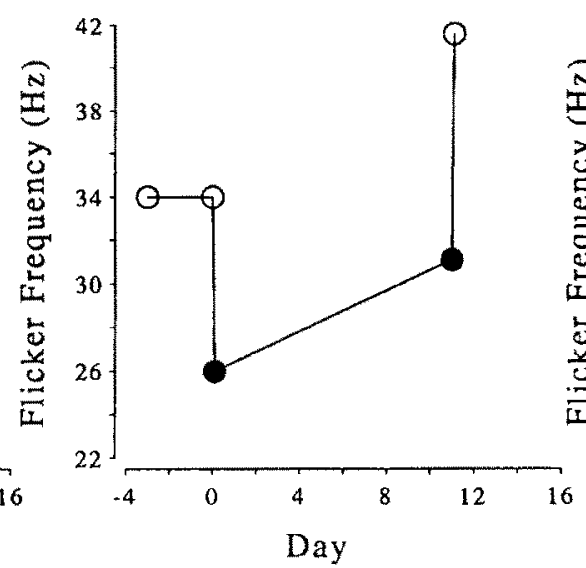

(d)

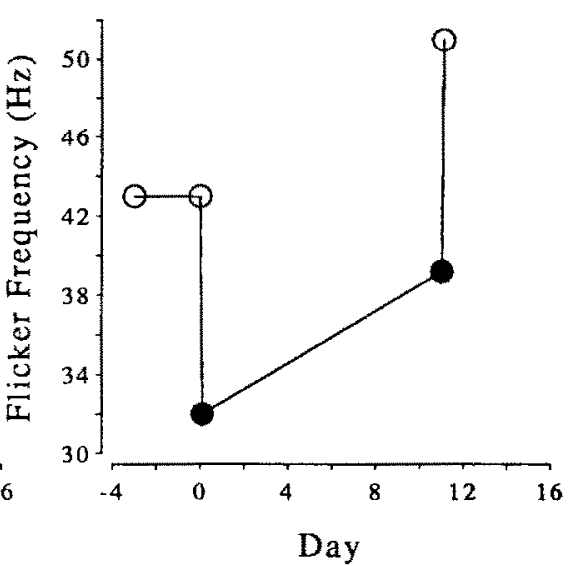

FIGURE 5. Results of simulations of Kelly's retinal model. (a) Simulation of the Pulfrich effect. (b)-(d) Simulations of the FFFs for $m$ values of $0.2,0.28$ and 0.84 respectively. The open circles represent simulations without the filter and the solid circles represent simulations with the filter in place.

but also demonstrates a significant overshoot in FFF on removal of the filter which was generally not seen in our results. The lack of overshoot in our FFF dala is a significant discrepancy from the model simulations and may indicate a dissociation in the mechanisms of the Pulfrich and FFF. The poor fit of the model may in part be due to the assumption that the Pulfrich effect can be entirely modelled by a change in the latency of the peak photoreceptor response. The effect of a reduced luminance not only changes this latency but also the shape of the response. Morgan $(1975,1977)$ has shown that a change in the shape of the photoreceptor response without a change in time-to-peak can be sufficient to generate the Pulfrich effect. So, if a moving object is viewed with a filter over one eye the impulse responses in each eye will have different shapes, leading to a complex correspondence problem. It is possible that the perception of the Pulfrich effect arises from the attempt to solve this correspondence task and that a change in the correspondence rule could lead to adaptation of the Pulfrich effect. However, a change in correspondence rule would not be expected to affect the FFF, which is presumably more directly related to the photoreceptor dynamics.
We chose to use Kelly's model of retinal processes as it allows us to model both the FFFs and impulse responses (Kelly, 1971). The model represents the full processing of the retina, rather than just phototransduction; however it is not easy to place physiological interpretations on the model parameters.

Our simulations confirm that the adaptation phase can be qualitatively explained by a change in only one parameter. This parameter corresponds to a "diffusion" rate parameter (Kelly, 1971). This process may therefore represent attenuation due to processes at one or more layers of the retina.

Lamb and Pugh (1992) have recently produced a model which accounts quantitatively for the activation steps involved in phototransduction in vertebrate photoreceptors. All the parameters in this model have physiological interpretations. Lamb and Pugh modelled the electrical responses secondary to the activation steps in the phototransduction cascade in response to brief flashes of light and to single photons. The electrical response at time $t>t_{\mathrm{cff}}$ to a brief flash of light was:

$$
1-\exp \left\{-\frac{1}{2} \phi\left[\frac{t-t_{\mathrm{efr}}}{\tau_{\mathrm{b}}}\right]^{2}\right\}
$$


where $\Phi$ is number of isomerizations of rhodopsin at $t=0, t_{\mathrm{eff}}$ is the effective pure time delay of transduction and $\tau_{\phi}$ is the characteristic time constant of transduction. Their model therefore contains two parameters which determined the time delays of transduction. The first is the pure delay $t_{\mathrm{efr}}$; this falls by about $2 \mathrm{msec}$ per $\log$ unit of intensity and therefore cannot explain the $16 \mathrm{msec}$ delay seen on placement of a $0.6 \mathrm{log}$ unit attenuating filter. The second is $\tau_{\phi}$ where $\tau_{\phi}{ }^{2}=v_{\mathrm{RP}} \beta_{\text {sub }} n$. These three parameters can be thought of as the gains of three separate processes: $v_{\mathrm{RP}}$ is the gain linking rhodopsin isomerization to the activation of phosphodiesterase (PDE) subunits, $\beta_{\text {sub }}$ is the hydrolytic gain linking activated PDE subunits to cyclic GMP concentration, and $n$ is the gain linking cyclic GMP concentration to channel activity. Theoretically a change in any of these could alter the time course of the response to match our data. Thus adaptation could change the value of $\tau_{\phi}$ thereby changing the slope of the electrical response. However, as this model does not include inactivation processes, it is not possible to model the flicker fusion data nor to find the latency of the peak of the impulse response as we have with Kelly's model.

The difference in interocular delay and in FFFs between the subjects is unlikely to be due entirely to phototransduction delays; there may be a secondary delay which differs between subjects. There may also be differences due to pupil size or dark adaptation state.

Although our data does not allow us to rule out cortical adaptation, we believe that the likely site of adaptation is the retina (probably the phototransduction process) and that it is the change in mean luminance levels which drives such a change. There is other evidence that suggest that mean luminance levels must change for adaptation to the Pulfrich effect and that the delay is peripheral. First, the Pulfrich effect can be seen in patients with unilateral optic neuritis secondary to multiple sclerosis (MS) and can be alleviated by filtering the normal eye (Rushton, 1975). The effect is due to slower than normal conduction in the demyelinated optic nerve. As these patients present with a significant Pulfrich effect some time after the likely demyelination it seems that they do not adapt to the effect (Rushton, 1975; Heron \& Dutton, 1989). These patients lack any mismatch in luminance between the two eyes. This would suggest that a change in mean luminance levels is essential for adaptation. Second, light deprivation studies in developing cats have shown that monocular lid suture causes a decrease in the visual latencies of geniculate X- and Y-cells (Sestokas \& Lehmkuhle, 1986), suggesting that lower mean luminance levels can indeed alter peripheral latencies. The adaptation to reduced luminance levels must be a process distinct from dark adaptation as its time-course of days does not correspond with the hours needed for complete dark adaptation. We also have direct evidence that dark adaptation was not a major contributor to the effects we saw, as there were only minor changes in interocular delay during the one hour of light adaptation following filter removal [see right hand side of Fig. 2(a)]. As a control for pupil size we examined the effects of wearing artificial pupils of $2.5 \mathrm{~mm}$ diameter on both eyes for the Pulfrich effect; this did not negate adaptation to the Pulfrich effect. Hence the results are not due to changes in pupil diameter

In conclusion, these experiments indicate that there are slow adaptive changes at least partly in the retina that affect photoreceptor latencies which are probably driven by long term monocular luminance levels. We do not need to postulate a cortical site of adaptation to explain our data.

\section{REFERENCES}

Baylor, D. A. \& Hodgkin, A. L. (1973). Detection and resolution of visual stimuli by turtle photoreceptors. Journal of Physiology, London, 234, 163-198.

Bolz, B., Rosner, G. \& Wassle, H. (1982). Response latency of brisk-sustained $(\mathrm{x})$ and brisk-transient $(\mathrm{y})$ cells in the cat retina. Journal of Physiology, London, 328, 171-190.

Carney, T., Paradiso, M. A. \& Freeman, R. D. (1989). A physiological correlate of the Pulfrich effect in cortical neurons of the cat. Vision Research, 29, 155 165 .

Cattell, J. M. (1986). The influence of the intensity of the stimulus on the length of the reaction time. Brain, 8, 512-515.

Cynader, M., Gardner, J. \& Douglas, R. (1978). Neural mechanisms underlying stereoscopic depth perception in cat visual cortex. In Cool, S. \& Smith, E. (Eds), Frontiers in visual science (pp. 373-386). Berlin: Springer.

Deno, D., Keller, E. \& Crandall, W. (1989). Dynamic neural network organization of the visual pursuit system. IEEE Transactions on Biomedical Engineering, 36, 85-92.

Douthwaite, W. \& Morrison, L. (1975). Flicker frequency and the Pulfrich phenomenon. American Journal of Optometry and Physiological Optics, 52. 745-749.

Drum, B. (1984). Cone response latency and log sensitivity: Proportional changes with light adaptation. Vision Research, 24 , 323-332

Enroth, C. (1952). The mechanism of flicker and fusion studied on single retinal elements in the dark adapted eye of the cat. Acta Physiologica Scandinavica (Suppl. 100), 27, 1-67.

Heron, G. \& Dutton, G. N. (1989). The Pulfrich phenomenon and its alleviation with a neutral density filter. British Journal of Ophthal mology, 73, 1004-1008.

Julesz, B. \& White, B. (1969). Short term visual memory and the Pulfrich phenomenon. Nature, 222, 639-641.

Kelly, D. (1971). Theory of flicker and transient responses I. Uniform fields. Journal of the Optical Society of America, 71, 537-546.

Kelly, D. (1972). Flicker. In Alpern, M. \& Hurvich, L. (Eds), Visual psychophysics, vol. 7(4) handbook of sensory physiology (pp. 273-302), Berlin: Springer.

Lamb, T. \& Pugh, E. (1992). A quantitative account of the activation steps involved in phototransduction in amphibian photoreceptors. Journal of Physiology, 449, 719-758.

Lennic, P. (1981). The physiological basis of variations in visual latency. Vision Research, 10, 815-824.

Lit, A. (1949). The magnitude of the Pulfrich stereophenomenon as a function of binocular differences of intensity at various levels of illumination. American Journal of Psychology, 62, 159-182.

Mansfield, R. J. W. \& Daugman, J. D. (1978). Retinal mechanisms of visual latency. Vision Research, 18, 1247-1260

Morgan, M. (1975). Stereoillusion based upon visual persistence Nature, 256, 639-640.

Morgan, M. (1977). Differential visual persistence between the two eyes: A model for the Fertsch-Pulfrich cffect. Journal of Experimental Psychology, 3, 484-495.

Morgan, M. J. \& Thompson, P. (1975). Apparent motion and the Pulfrich effect. Perception, 4, 318 
Pulfrich, C. (1922). Die stereoskopie im dienste der isochromen und heterochromen photometrie. Naturwissenschaften, 10,553-564.

Regan, D., Bevereley, K. \& Cynader, M. (1979). Stereoscopic systems for position in depth and for motion in depth. Proceedings of the Royal Society of London, B, 204, 485-501.

Rogers, B. J. \& Anstis, S. M. (1972). Intensity versus adaptation and the Pulfrich stereophenomenon. Vision Research, 12, 909-928.

Roufs, J. (1972). Dynamic properties of vision-II. Theoretical relationship between flicker and flash thresholds. Vision Research, 12, 279-292.

Rushton, D. (1975). Use of the Pulfrich pendulum for detecting abnormal delay in the visual pathway in multiple sclerosis Brain, 98 . 283-296.

Sestokas, A. K. \& Lehmkuhle, S. (1986). The effects of monocular deprivation on the visual latency of geniculate $x$ - and $y$-cells in the cat. Developmental Brain Research, 30, 93-95.

Watson, A. \& Pelli, D. (1983). QUEST: A Bayesian adaptive psychometric method. Perception and Psychophysics, 33, 113120

Acknowledgements - This research was supported by the Wellcome Trust and the Medical Research Council. We would like to thank John Stein, Michael Morgan, David Regan, Tony Watts and especially Andrew Parker for their helpful suggestions.

\section{APPENDIX}

Many models have been developed to explain both flicker fusion and temporal impulse response data (Kelly, 1971; Roufs, 1972). Most are based on the schematic diagram in Fig. 6 (Kelly, 1972).

Here, $f^{\prime}(t)$ represents the stimulus waveform (in units of td) and $h(t)$ represents the output waveform at some point in the retina or central nervous system. Usually the control system is regarded as obeying the law of superposition (the output to the sum of two given inputs is equal to the sum of the output to the inputs presented singly) and can therefore be modelled with a linear transfer function $G(\omega)$ ). Mos systems can be approximated as linear over some small range of inputs. The output of the model then passes to a detector. This detector possesses a threshold for the detection of flicker and therefore cannot be linear. For example, Kelly models the detector as responding to the peak-to-trough amplitude of the output $h(t)$; flicker being reported when this output amplitude exceeds a certain fixed threshold. The models mainly differ in the form of $G(\omega)$. The amplitude of this transfer function alone is enough to determine flicker thresholds. However to predict impulse responses both the amplitude and phase are needed.

The first component of Kelly's model is governed by a linear process believed to represent attenuation at one or more layers in the retina. This stage represents a low pass filter diffusion process and therefore limits the high frequency component of the flicker fusion curves. It can be approximated as the transfer function $G_{1}(s)$ in which $\tau$ is the time constant of the diffusion process.

$$
G_{1}(s)=C \mathrm{e}^{[-12 \tau, y) ! z]} .
$$

The second stage is a high pass filter based on models of lateral inhibition in the retina which include feedback with a low pass filter As a basic element of this network Kelly chose a simple RC integrator with time constant $2 \pi / \alpha$ and gain $K$. He placed such a unit

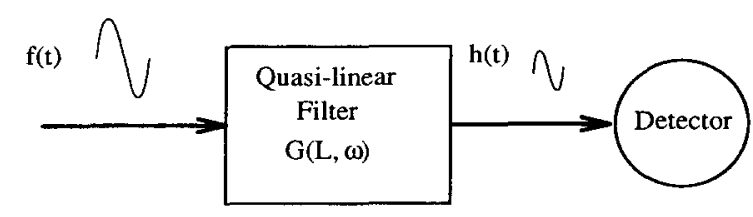

FIGURE 6. Schematic representation of a flicker sensitivity model. Input $f(t)$ passes through a quasi-linear model (linear for any single value of luminance $L$ ). Output $h(t)$ then passes to a flicker detector which determines whether flicker is present.
$K(L) /(s+\alpha)$ in a negative feedback pathway. Each unit consisted of multiple feedback loops and each loop could contain a variable number of filters. This produced a transfer function, for a given luminance stimulus $L$, of the following form:

$$
G_{2}(s)=\left[\frac{(s+\alpha)^{2}}{(s+K)^{2}+K^{2}}\right]^{r^{2}} .
$$

The parameter $r$ represents the number of inhibiting stages in the cascade. Rather than use a non-linear model Kelly replaced this stage by a linear one whose parameters depend only on background luminance $L . K$ and $r$ therefore vary with luminance level: $K$ varies approximately as the 0.4 power of $L$ whereas $r$ varies with its logarithm. $\alpha$ is however constant. The overall transfer function of the system is therefore

$$
G(s)=G_{1}(s) G_{2}(s)=C\left[\begin{array}{c}
(s+x)^{2} \\
(s+K)^{2}+K^{2}
\end{array}\right]^{r 2} \mathrm{e}^{\mid-(2, s) ! ? !} .
$$

The combined magnitude response which represents the amplitude response to a $1 \mathrm{td}$ amplitude input for a particular frequency $\omega$ is

$$
|G(j \omega)|=C\left[\frac{\left.(\omega)^{2}+\alpha^{2}\right)^{2}}{\omega^{4}+4 K^{4}}\right]^{r \cdot 4} \text { el }
$$

Therefore taking an inpul sinusoid with anplitude $L m$ fron equation (1) produces an output with amplitude $L m|G(\omega)|$ for a frequency $\omega$. Taking a detector with an arbitrary threshold of one, the theoretical FFF for given values of $m$ and $L$ can be found as the value of $\omega$ which satisfies:

$$
m L|G(j())|=1 .
$$

In order to simulate the results from our experiment, we measured the latency of the peak of the impulse response of Kelly"s model. The interocular time delay was then taken as the difference in latencies between the two eyes. We therefore simulated the impulse response of the model by calculating the real and imaginary parts of $G(j \omega)$ for frequencies below $100 \mathrm{~Hz}$ and used an inverse fast Fourier transform to estimate the impulse response numerically.

The aim of the simulations was to reproduce qualitatively our results for both the flicker fusion and Pulfrich experiments. In particular we wished to be able to model the adaptation period by altering only one parameter in the model. The change of this chosen parameter should lead to both appropriate increases in the FFFs for the three $m$ values and a decrease in the latency of the peak of the impulse response. A change in $C$ alone has no effect on the latency of the impulse response but only scales its magnitude [and therefore can scale the arbitrary threshold level assumed in equation (6)]. Changes in $\alpha$ were found to have very little effect on the flicker frequencies; these two parameters could therefore not reproduce the adaptation seen. To achieve an increase in flicker frequency $K$ needed to decrease, but this also led to an increase in latency of the impulse response rather than a decrease. Only an increase in $r$ or a decrease in $\tau$ could both explain the increase in flicker frequency and decrease in impulse latency. However our simulations demonstrated that a change in $r$ that reduced the impulse response latency sufficient to model the Pulfrich adaptation produced a non-significant change in the FFFs. Thus only changes in $\tau$ seemed appropriate.

We chose to use the parameter values published in Kelly's (1971) paper. We therefore set $1 / C=0.00018 \mathrm{td}$ and $\alpha=11 \mathrm{rad} \mathrm{sec}^{-1}$ and these remained fixed for all simulations. However Kelly's paper dealt with uniform field stimuli for flicker whereas we studied foveal stimuli. The FFFs for such a stimulus are known to be lower than peripheral stimuli. We therefore needed to select a value for $L$, the luminance, which was lower than actually measured, so that the FFFs of the simulation were in the same range as our results. Thus for simulations without the filter we used Kelly's values of $L=7.5 \mathrm{td}, K=2 \pi 13.1$ and $r=3.3$. For the filter condition ( $75 \%$ attenuating) we interpolated between Kelly's published values and used $L=1.75(25 \%$ of prefilter value), $K=2 \pi 9$ and $r=3.0$. For each of the four conditions we calculated the FFFs for the three values of $m$ and also the difference between the latency of the peak of the impulse response of the attenuated and unattenuated eye. 
Model simulations

Figure 5 shows the results of the model simulations. For the pre- and post-filter placement on day $0, \tau=0.5$. Reducing the luminance by $75 \%$ resulted in an increase in the impulse response latency of $9 \mathrm{msec}$. Then by adjusting $\tau$ to 0.42 , to simulate an adaptive change in the retina, this latency reduced to 2 msec. Returning the luminance to its original value caused an overshoot of $5 \mathrm{msec}$ Simulations of FFFs were similar for all three values of $m$ with the FFF initially falling by about $11 \mathrm{~Hz}$ on placement of the filter, rising to nedar prefilter values as $\tau$ fell to 0.42 and then overshooting. 